\title{
An experimental study of an efficient supercapacitor stacking scheme to power mobile phones
}

\author{
José Monteiro \\ Instituto Universitário de Lisboa (ISCTE-IUL) \\ Lisboa, Portugal \\ jjnmonteiro@gmail.com
}

\author{
Nuno Garrido \\ Instituto Universitário de Lisboa (ISCTE-IUL), \\ Instituto de Telecomunicações (IT-IUL) \\ Lisboa, Portugal \\ nuno.garrido@iscte.pt
}

\author{
Ricardo Fonseca \\ Instituto Universitário de Lisboa (ISCTE-IUL) \\ GoLP / Instituto de Plasmas e Fusão Nuclear \\ Lisboa, Portugal \\ ricardo.fonseca@iscte.pt
}

\begin{abstract}
This paper presents an implementation of a solution for efficiently using supercapacitors to power mobile phones. This system achieves an increase in the autonomy of the device by maximizing the energy harnessed from the supercapacitors. Test results confirm the uplift in the device's autonomy by using a supercapacitor stacking scheme, when comparing with the use of a DC/DC voltage converter alone. A specific charging scheme for this solution is also prototyped, allowing ultrafast and incremental charges without affecting the scheme's operability.
\end{abstract}

Keywords - supercapacitors; mobile phones; energy efficiency; fast charging

\section{INTRODUCTION}

Mobile phones, as many other portable electronic devices powered by electrical energy, generally use electro-chemical Li-ion batteries to store energy. These batteries are light and can come in many different shapes and sizes, being easily adapted to provide the required autonomy to our everyday portable electronics. However, they exhibit lengthy charging cycles and a short lifespan.

A relatively recent, alternative technology to store electrical energy is the electric double-layer capacitor (EDLC), also known as the supercapacitor. Supercapacitors are already being used in many different applications combined with batteries, occasionally even replacing them. The ability of the supercapacitor to charge and discharge much faster than the battery, combined with its long lifespan, is shown to improve the performance of some electronic systems [1]. For the time being, the energy density of the supercapacitor makes its usage in mobile electronic devices like the mobile phone unviable, if the same autonomy is desired. However, research studies within this particular property [2] are revealing that it could be improved in the near future. Notwithstanding, their high power density can somehow compensate for this, as the mobile device can be charged more times a week but for a much shorter period.
As mobile phones are designed to operate from a fixed DC voltage source, the main technical difficulty lies in matching the non-fixed supercapacitor cell voltage to the required 3.7V. Reference [3] proposes a solution to power mobile phones, combining the use of a DC/DC converter with a supercapacitor stacking scheme, assuring that most of the energy stored in the supercapacitors is usable, whilst taking the most of the DC/DC converter's efficiency. This paper intends to experimentally validate that solution. Some particular aspects of the implementation are shown, namely the type of used switches, a key component for the success of this scheme. Measured results confirm the scheme's effectiveness in increasing the mobile phone's autonomy either in standby and call modes. A charging scheme for the system is also implemented and tested, showing that this solution is compatible with fast and incremental charges.

\section{USABLE ENERGY IN SUPERCAPACITORS}

A mobile phone is usually designed to operate from a DC voltage source, typically a battery with a nominal voltage of $3.7 \mathrm{~V}$. In order to use supercapacitors for this purpose, a solution that solve the problems of 1) the low maximum supercapacitor cell voltage and 2) the voltage dependency on the amount of charge the supercapacitor is holding $(V=Q / C)$, is the use of a DC/DC step-up converter. Considering this solution and letting $V_{\mathrm{C}(\mathrm{MAX})}$ be the maximum voltage across the supercapacitor and $V_{\mathrm{IN}(\mathrm{MIN})}$ the minimum input voltage for the step-up converter to operate, the usable energy $U E$ of the supercapacitor is given by:

$$
U E=E_{\mathrm{HARNESSED}} / E_{\mathrm{TOTAL}}=1-\left(V_{\mathrm{IN}(\mathrm{MIN})} / V_{\mathrm{C}(\mathrm{MAX})}\right)^{2}
$$

Considering that the typical maximum voltage of a supercapacitor cell is $2.7 \mathrm{~V}$, the usable energy can only be above $90 \%$ when a boost converter with a minimum operating input voltage below $0.86 \mathrm{~V}$ is used. Although some 
manufacturers have step-up converters with this characteristic, they cannot provide the required output current for the mobile phone under these circumstances. Moreover, the converter's efficiency is lower when it is operating within the low input voltage range. To overcome these problems, [3] proposes the use of a voltage controlled supercapacitor stacking scheme along with the step-up converter, as shown in Fig. 1.

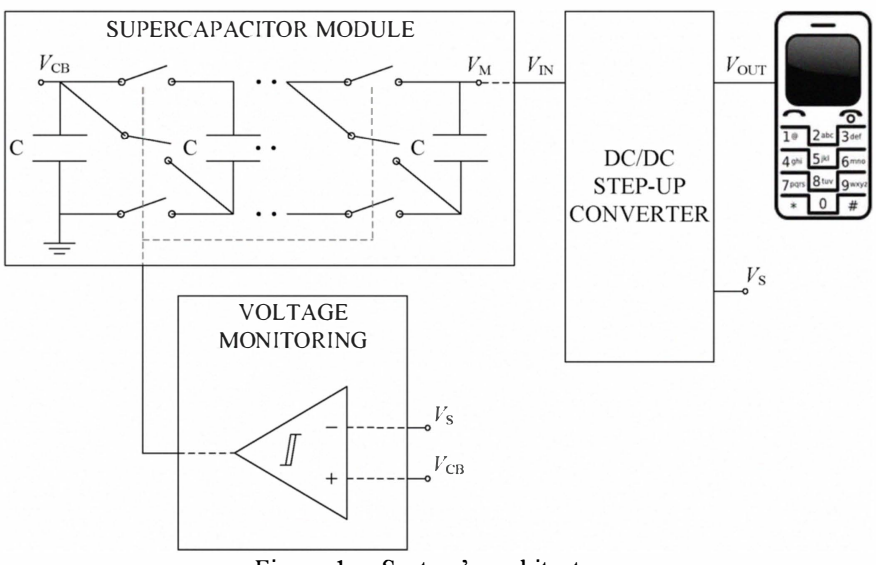

Figure 1. System's architecture

With this scheme, the output voltage of the supercapacitor module $V_{\mathrm{M}}$ can be doubled by stacking half of the cells on top of the other half, when $V_{\mathrm{M}}$ drops below the switching voltage $V_{\mathrm{S}}$. This procedure can be done $N$ times for a supercapacitor module composed by $2^{N}$ cells, where the usable energy is given by:

$$
U E=1-\left[\left(V_{\mathrm{IN}(\mathrm{MIN})} / 2^{N}\right) / V_{\mathrm{C}(\mathrm{MAX})}\right]^{2}
$$

As shown by (2), the usable energy is not directly dependent on the switching voltage, therefore $V_{\mathrm{S}}$ can be adjusted so that the step-up converter operates within its highest efficiency input voltage range.

\section{PROTOTYPING THE SUPERCAPACITOR STACKING SCHEME}

The presented circuit was implemented using Linear Technology's LTC3402 as the DC/DC step-up converter, since it is a high efficiency voltage converter which can operate with minimum input voltages below $1.0 \mathrm{~V}$. The voltage monitoring block was implemented using the LTC1841 ultralow power comparator, to make the Schmitt-triggered comparators, therefore minimizing the impact of this block in the overall consumption. The supercapacitor module was designed for a total capacitance $C_{\mathrm{T}}$ of $100 \mathrm{~F}$ composed by four $25 \mathrm{~F}$ EDLCs (either for $N=0,1$ and 2). As initially all the cells are charged at a $V_{\mathrm{C}}$ voltage of approximately $2.7 \mathrm{~V}$, the total energy $E_{\mathrm{T}}$ of the supercapacitor module is given by (3). A total energy of $101.25 \mathrm{mWh}$ is obtained, which is, in approximately the same volume, 1/30 of the total energy stored in a Li-ion battery of a typical mobile phone.

$$
E_{\mathrm{T}}=\frac{1}{2} C_{\mathrm{T}} V_{\mathrm{C}}^{2}
$$

Although Fig. 1 depicts the supercapacitor module using single-pole single-throw (SPST) switches, the prototype uses single-pole double-throw (SPDT) switches. This approach, not only reduces the number of switches by $1 / 3$ but also avoids the need for additional circuitry to invert the logic of the control signal for the switches, once some are closed while others are opened. The implemented circuits for $N=1$ and $N=2$ are depicted in Fig. 2 and in Fig. 3, respectively.

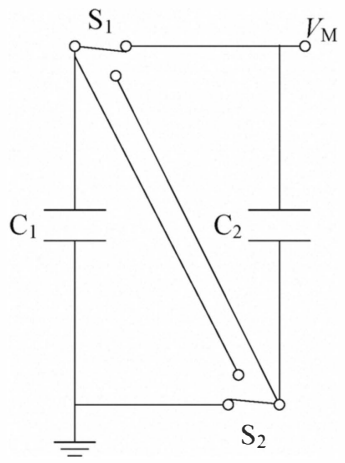

Figure 2. Implemented supercapacitor module for $N=1$

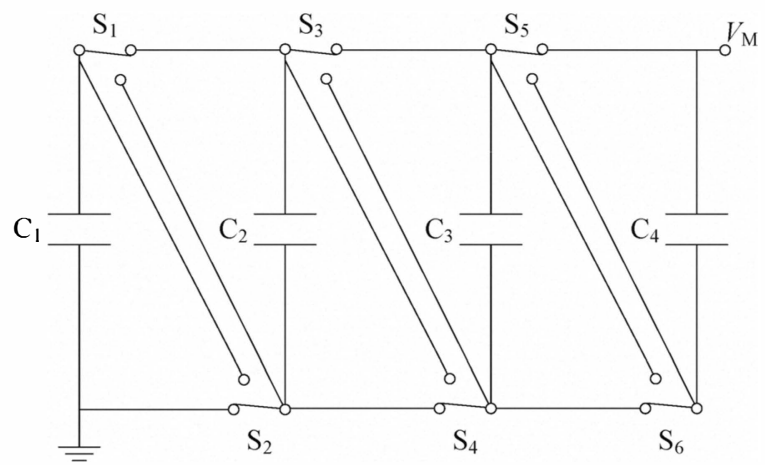

Figure 3. Implemented supercapacitor module for $N=2$

With this specific implementation, when a stacking operation occurs and the cells are put in series, the switches responsible for the stacking are both conducting current to the output, reducing to half the impact of their on-resistance $R_{\mathrm{ON}}$. Fig. 4 and Fig. 5 schematically represent the switches' on-resistance, as well as the currents that pass through them, in all possible topologies for $N=1$ and $N=2$, respectively, assuming that all the switches have the same $R_{\mathrm{ON}}$ and all the supercapacitors are identical.
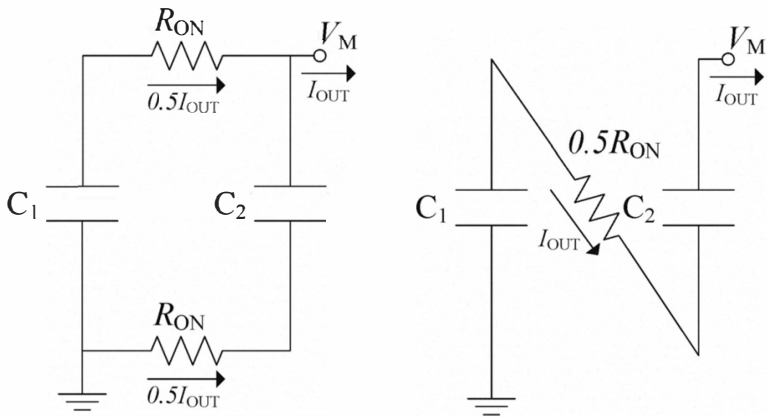

Figure 4. Supercapacitor module schematic for $N=1$ showing the value for the switches' $R_{\mathrm{ON}}$ for different topologies 

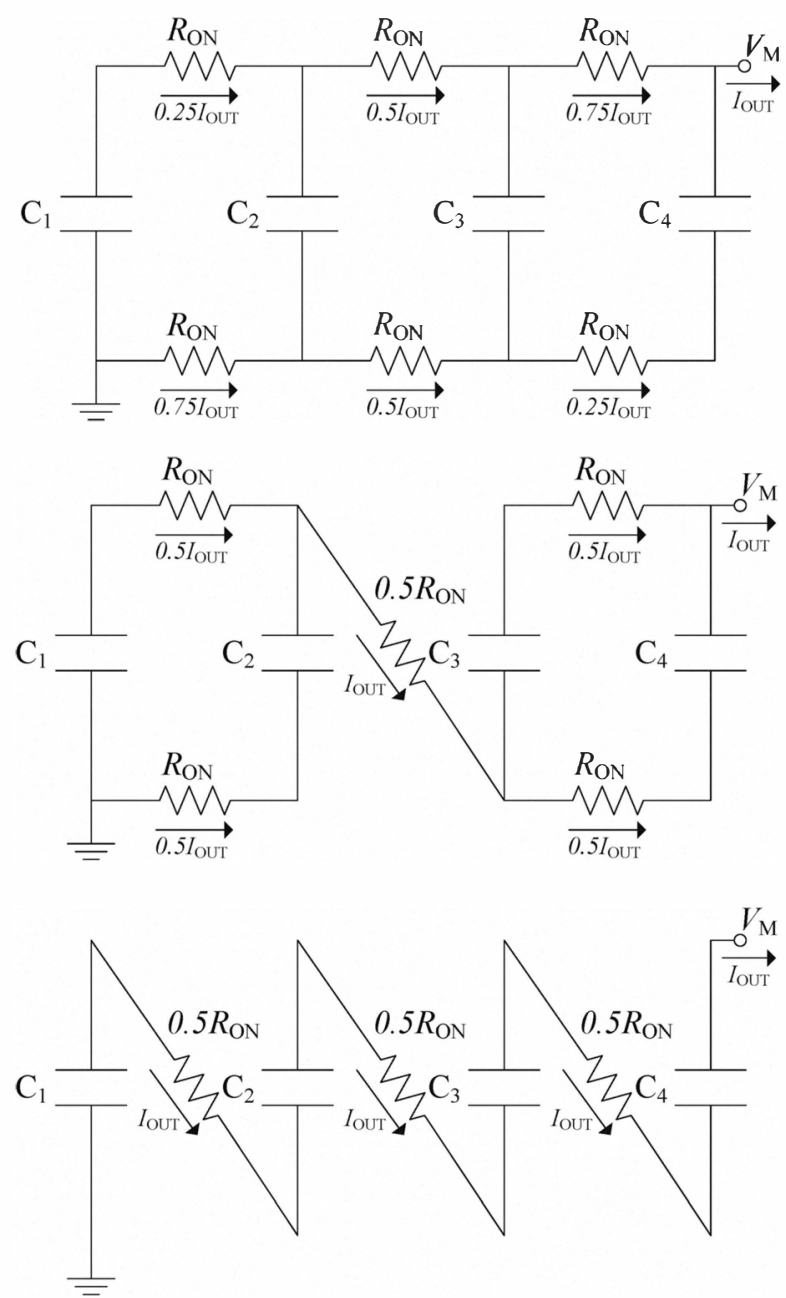

Figure 5. Supercapacitor module schematic for $N=2$ showing the value for the switches' $R_{\mathrm{ON}}$ for different topologies

\section{TEST RESULTS}

A Nokia 3310 mobile phone was used for the tests, fed by the boost converter's output, which was adjusted to a fixed voltage of approximately $3.7 \mathrm{~V}$.

Fig. $6^{1)}$ shows the typical behavior of input $V_{\mathrm{IN}}$ and output $V_{\text {OUT }}$ voltages of the step-up converter for $N=0,1$ and 2 in standby mode. As expected, the mobile phone's autonomy is increased in $N=1$ and $N=2$, when comparing with $N=0$. In these cases $(N=1$ and $N=2), V_{\text {OUT }}$ shows no significant disturbance when the switching occurs and the topology of the supercapacitor module is changed, therefore not affecting the mobile phone's operation. We also observe that the supercapacitor module discharges faster for lower $V_{\mathrm{IN}}$ voltages since, in this case, the boost converter needs to consume higher currents to maintain the output voltage fixed.

The overall average results of the performed tests in standby mode are presented in Table $\mathrm{I}^{2)}$. These results show a considerable improvement in the device's autonomy when using the stacking scheme, either for $N=1(16.9 \%)$ and $N=2$ $(19.6 \%)$. The amount of charge drawn to the mobile phone is also increased for $N=1(15.5 \%)$ and $N=2(16.7 \%)$. Although

\footnotetext{
1) Although several tests for each value of $N$ were made, the results shown in Fig. 6 and Fig. 7 represent only one test for each value of $N$, thus further conclusions concerning autonomy gains cannot be taken from these data alone

2) Before each testing, the four supercapacitors were charged at $500 \mathrm{~mA}$ and left at $2.7 \mathrm{~V}$ for several hours so that the energy losses due to leakage currents would be negligible
}
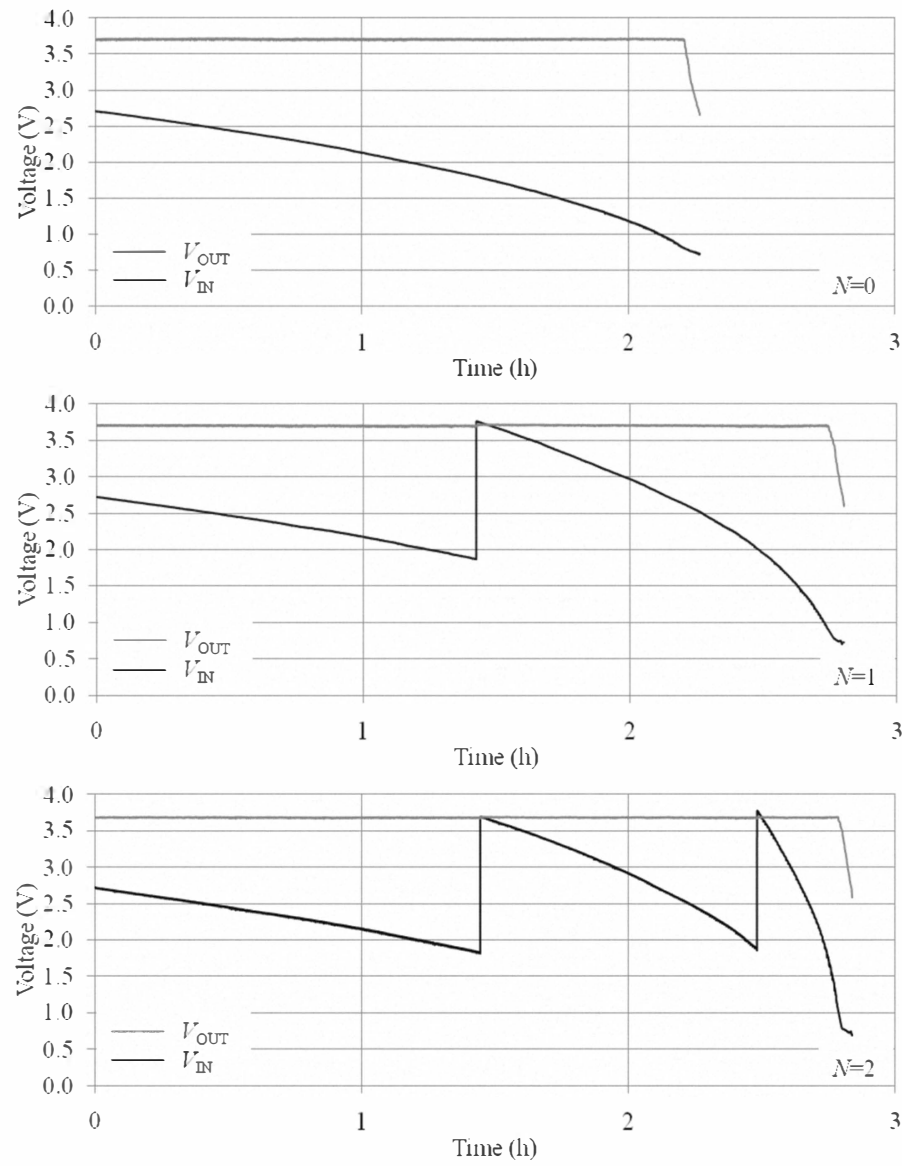

Figure 6. $\quad V_{\mathrm{IN}}$ and $V_{\text {OUT }}$ from the converter for $N=0,1$ and 2 in standby mode

TABLE I. MEASURED RESULTS FOR STANDBY MODE

\begin{tabular}{|c|c|c|c|c|c|}
\hline \multirow{2}{*}{$\boldsymbol{N}$} & \multirow{2}{*}{$\begin{array}{c}\text { Avg. } \\
\boldsymbol{I}_{\text {LOAD }} \\
\end{array}$} & \multicolumn{2}{|c|}{ Charge $^{\mathrm{a}}$} & \multicolumn{2}{c|}{ Autonomy } \\
\cline { 3 - 6 } & $(\mathbf{m A})$ & $(\boldsymbol{C})$ & Gain $^{\mathrm{b}}$ & (h) & Gain $^{\mathrm{b}}$ \\
\hline $\mathbf{0}$ & 7.31 & 52.6 & $\mathrm{n} / \mathrm{a}$ & 2.02 & $\mathrm{n} / \mathrm{a}$ \\
\hline $\mathbf{1}$ & 7.33 & 60.7 & $15.5 \%$ & 2.36 & $16.9 \%$ \\
\hline $\mathbf{2}$ & 7.28 & 61.3 & $16.7 \%$ & 2.42 & $19.6 \%$ \\
\hline
\end{tabular}

a. Average current, and amount of charge drawn to the load (i.e. the mobile phone)

b. Gains are relative to $N=0$

smaller, the difference in the device's autonomy between $N=1$ and $N=2$ is still noticeable. These results confirm the effectiveness of the stacking approach, while suggesting that the additional circuitry needed to implement the supercapacitor module for $N=2$ may also be worthwhile.

The typical behavior of $V_{\mathrm{IN}}$ and $V_{\mathrm{OUT}}$ in call mode for $N=0,1$ and 2 is depicted in Fig. $7^{1)}$. For $N=1$ and $N=2$ we can observe some oscillations in $V_{\mathrm{IN}}$, which become relatively high for $N=2$. This is due the fact that, during a call, the mobile phone's current consumption is highly unstable, oscillating from tens to hundreds of milliamps. Therefore, since the on-resistance of the switches that were used is not negligible at higher currents, the supercapacitor module voltage $V_{\mathrm{M}}$ (or $V_{\mathrm{IN}}$ ) is lowered as the current increases. This phenomenon also causes disturbances in the output voltage, however, this does not reveal to affect the mobile phone's operability. 

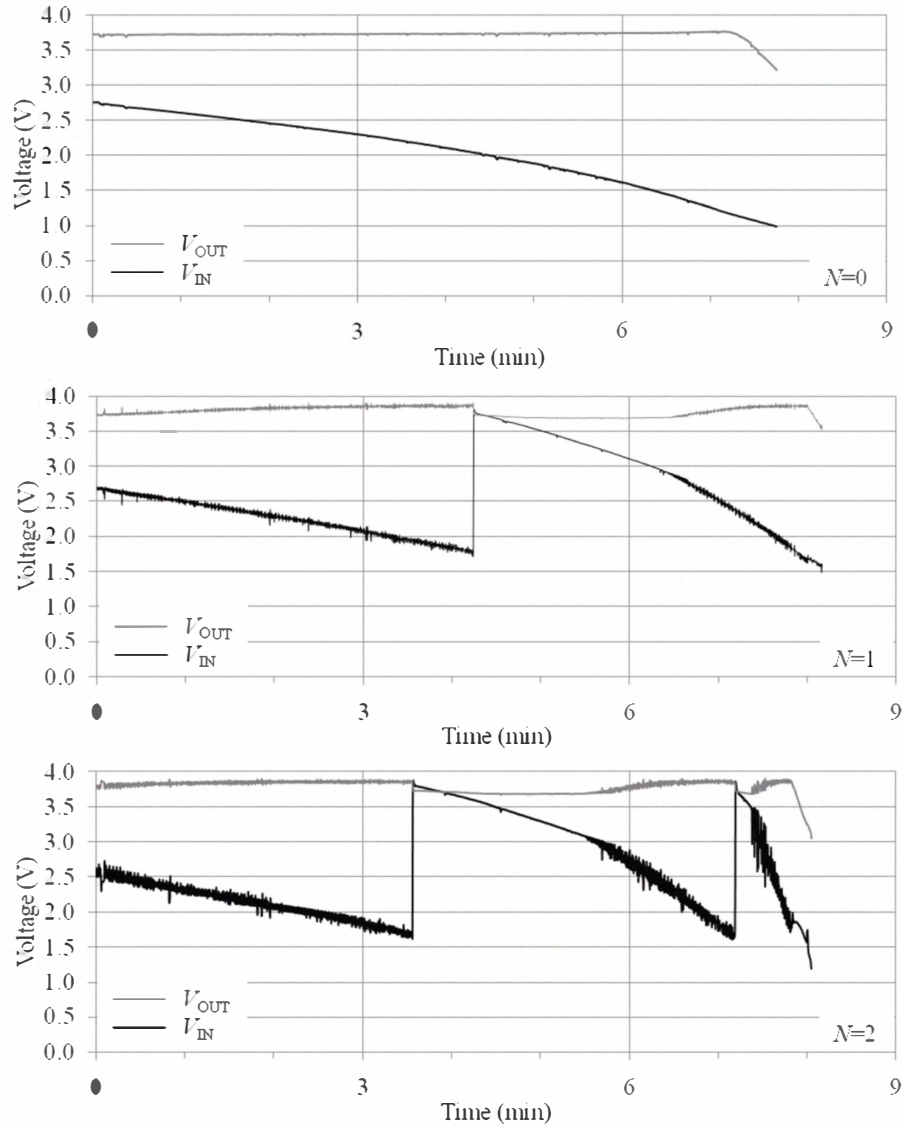

Figure 7. $V_{\mathrm{IN}}$ and $V_{\text {OUT }}$ from the converter for $N=0,1$ and 2 in call mode

The overall average results of performed tests in call mode are summarized in Table $\mathrm{II}^{2)}$, where we can observe a clear improvement in the mobile phone's autonomy for $N=1(7.8 \%)$ and $N=2(8.4 \%)$ when comparing with the case of not using the stacking approach $(N=0)$. While the autonomy slightly increases from $N=1$ to $N=2$, the amount of charge drawn to the mobile phone decreases. As this can somehow be contradictory, it can also suggest that with a bigger amount of performed tests the results for $N=1$ and $N=2$ would converge to approximately the same value. Considering the simulation results on the impact of the switches' on-resistance presented in [3], these gains can be uplifted if lower $R_{\mathrm{ON}}$ switches are used.

TABLE II. MEASURED RESULTS FOR CALL MODE

\begin{tabular}{|c|c|c|c|c|c|}
\hline \multirow{2}{*}{$N$} & \multirow{2}{*}{$\begin{array}{c}\text { Avg. } \\
I_{\text {LOAD }} \\
(\mathbf{m A})\end{array}$} & \multicolumn{2}{|c|}{ Charge $^{a}$} & \multicolumn{2}{|c|}{ Autonomy } \\
\hline & & (C) & Gain $^{b}$ & $(\min )$ & Gain $^{\mathrm{b}}$ \\
\hline 0 & 118.3 & 52.7 & $\mathrm{n} / \mathrm{a}$ & 7.43 & $\mathrm{n} / \mathrm{a}$ \\
\hline 1 & 117.9 & 56.6 & $7.4 \%$ & 8.00 & $7.8 \%$ \\
\hline 2 & 116.2 & 56.1 & $6.4 \%$ & 8.05 & $8.4 \%$ \\
\hline
\end{tabular}

a. Average current, and amount of charge drawn to the load (i.e. the mobile phone)

b. Gains are relative to $N=0$

\section{CHARGING THE SUPERCAPACITOR MODULE}

In this supercapacitor stacking scheme the cells are not always connected to the same nodes due to the stacking of the cells. This way, charging all the cells in a supercapacitor module with $N>0$ is not as simple as charging a module that is not using the stacking scheme $(N=0)$.

A possible solution to charge the supercapacitor module for $N>0$ is proposed in [3], where a supercapacitor charger is connected to each cell in the module, enabling parallel charging whilst avoiding that the charging currents pass directly through the switches and dissipate power. However, for this solution to work correctly, the chargers must be fed by individual differential output DC voltage sources, since they need to operate from distinct, varying voltage levels. To overcome this issue, this paper proposes the scheme shown in Fig. 8.

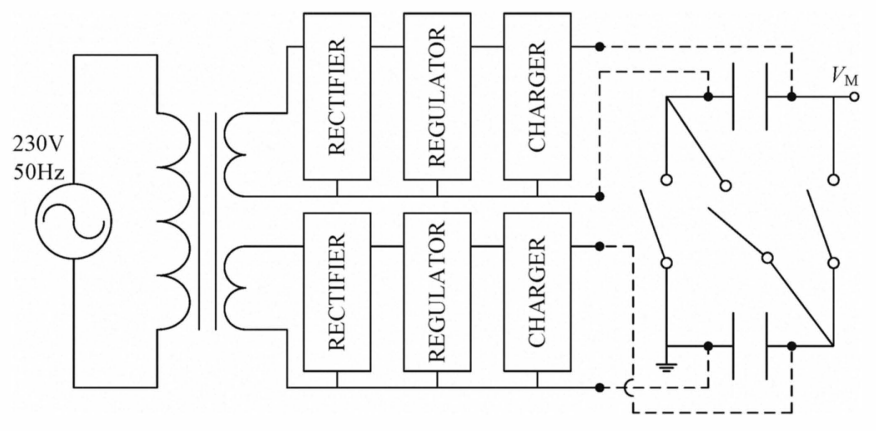

Figure 8. Implementation of the supercapacitor module charging scheme for $N=1$

This scheme shows an AC supplied charger for the mobile phone using a supercapacitor module with $N=1$. The need for a different floating ground for each charger is solved by using a transformer with as many secondary windings as chargers needed in the design, as this guarantees electrical isolation between the DC voltage sources.

Fig. 9 and Fig. 10 show the input $V_{\mathrm{IN}}$ and output $V_{\mathrm{OUT}}$ voltages of the step-up converter along with the voltage $V_{\mathrm{CB}}$ and charging current $I_{\mathrm{CB}}$ of the bottom cell of the module, when charging the supercapacitor module for $N=1$ and $N=2$, respectively. For both situations the charging was made at $1.5 \mathrm{~A}$ per each $25 \mathrm{~F}$ of capacitance, thus making a total charging current of $6 \mathrm{~A}$.

For $N=1$ the charging started when the cells in the module are in series topology. Therefore, we can observe the unstacking of the cells back to parallel connection when the bottom supercapacitor voltage $V_{\mathrm{CB}}$ goes above the switching voltage $V_{\mathrm{S}}$. For $N=2$ the charging also started when all cells were stacked. Contrarily to what is observed when the module is discharging, the bottom cell voltage $V_{\mathrm{CB}}$ is constantly increasing (as well as the voltage across each cell), whilst the input voltage of the boost converter $V_{\mathrm{IN}}$ is reflecting the topology changes in the module.

Although the charging can be fast, the supercapacitors should be kept at the charging voltage for a longer period of time, in order to minimize the energy losses due to leakage currents.

This parallel charging scheme is proven to allow fast charges without compromising the supercapacitor module or the system's integrity, namely the switches. The amount of current used to charge each cell $(1.5 \mathrm{~A})$ is far from the 


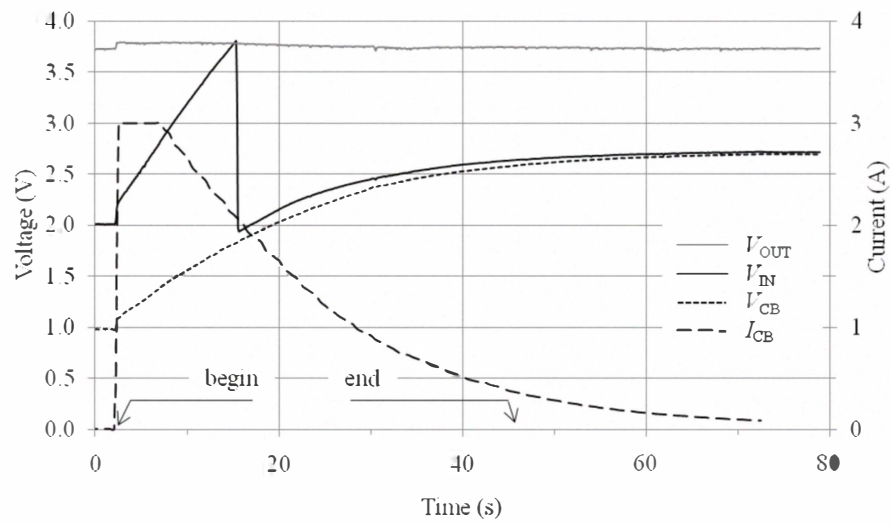

Figure 9. $V_{\mathrm{IN}}, V_{\mathrm{OUT}}, V_{\mathrm{CB}}$ and $I_{\mathrm{CB}}$ during the charge $(N=1)$

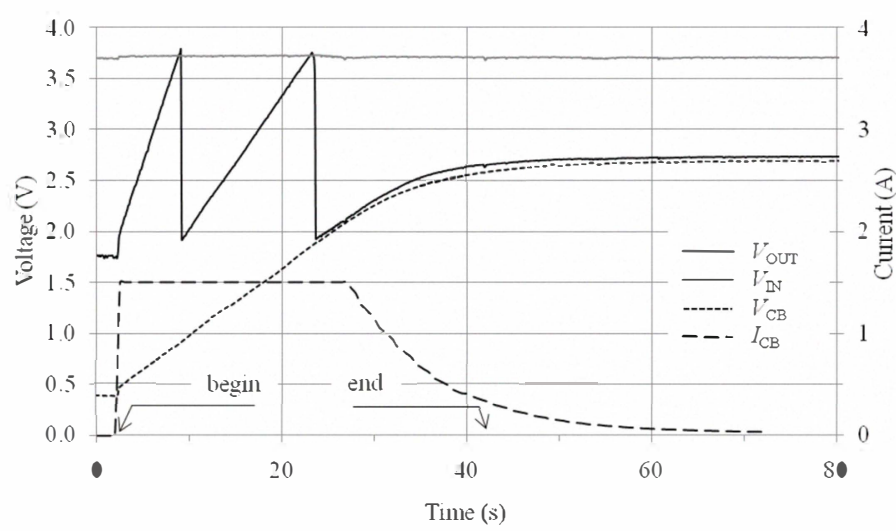

Figure 10. $V_{\mathrm{N}}, V_{\mathrm{OUT}}, V_{\mathrm{CB}}$ and $I_{\mathrm{CB}}$ during the charge $(N=2)$

maximum allowed current of the used supercapacitors, which is rated at 16.9A. Thus, theoretically, this particular module could be charged more than ten times faster, taking full advantage of the supercapacitor's power density.

\section{CONCLUSIONS}

The supercapacitor stacking scheme presented in [3] and experimentally tested in this paper, reveals effective improvements in the autonomy gains either for standby or call modes when compared with the use of a DC/DC step-up voltage converter alone. Although the resulting autonomy improvement from the first stacking operation is undoubtedly worthwhile, the second stacking operation reveals only a slightly improvement when compared to the first one. This way, when designing the system, the number of stacking operations must be complacent with the maximum allowed complexity for the device.

The presented values are a result of the combination of all the general-purpose components that were used. Therefore, higher autonomy gains could be obtained by optimizing some characteristics of the system, namely 1) reducing the switches on-resistance and 2) using an application specific DC/DC step-up converter.

The prototyped solution for a parallel charging of the supercapacitor module shows to be compatible with high current charges, without compromising the system's operability, therefore taking advantage of the supercapacitor's high power density.

Although, for the time being, supercapacitors have a relatively low energy density to outdo the present role of the battery, its advantages can, in a near future, change the paradigm of charging mobile phones.

\section{ACKNOWLEDGMENT}

The authors thank Vina Technology Co., Ltd and CAP-XX Ltd for all the support.

\section{REFERENCES}

[1] A.Schneuwly, R. Gallay, "Properties and applications of supercapacitors: from the state-of-the-art to future trends", Proceedings of PCIM, 2000

[2] R. Signorelli, D. Ku, J. Kassakian, J. Schindall, "Electrochemical double-layer capacitors using carbon nanotube electrode structures", Proceedings of the IEEE, vol. 97, No. 11, November 2009

[3] J. Monteiro, N. Garrido, R. Fonseca, "Efficient supercapacitor energy usage in mobile phones", IEEE ICCE-Berlin, 2011, in press 